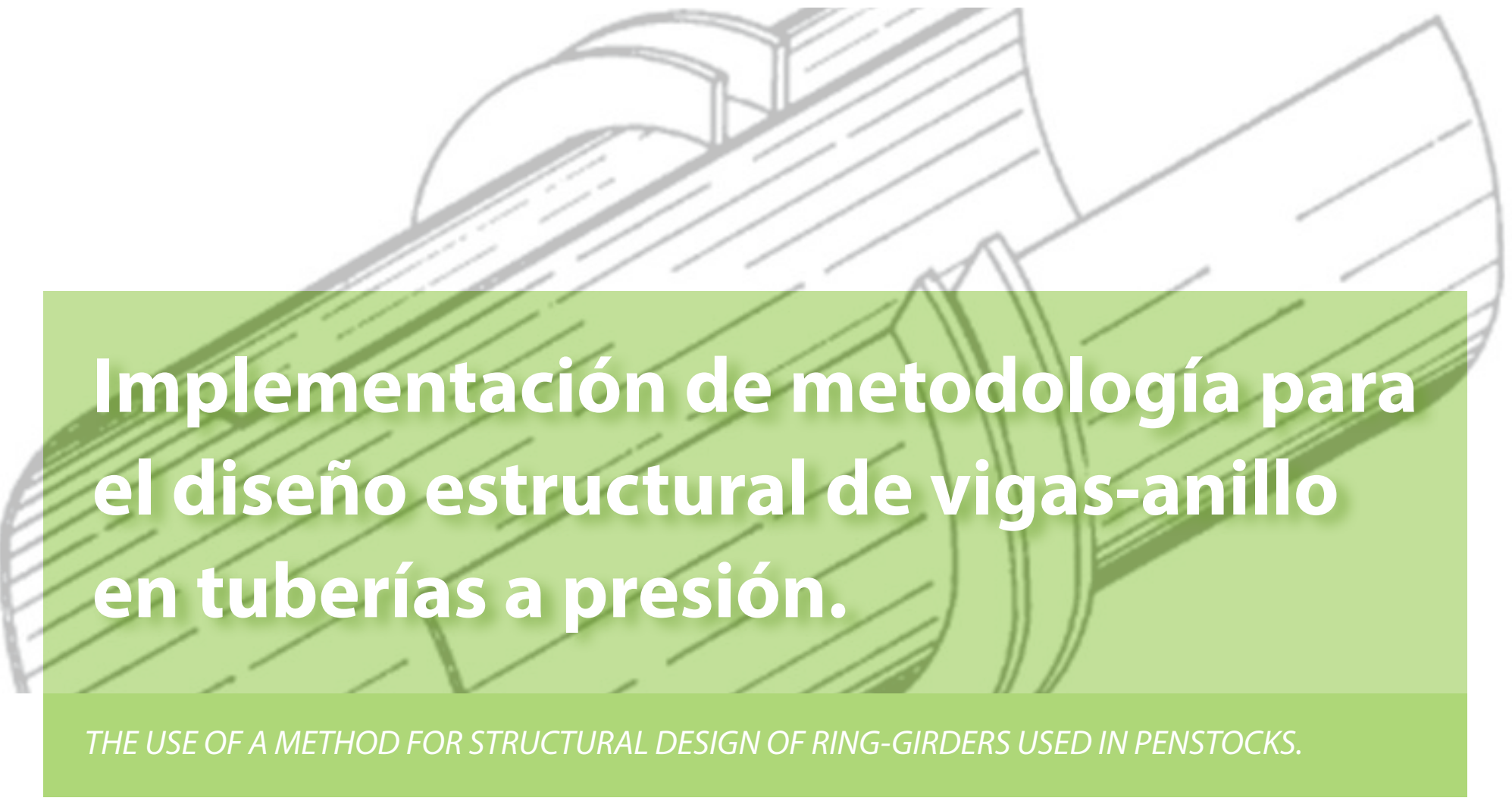

\section{Mónica Aguilera Chaves}

Estudiante Ingeniería Civil, Universidad de Costa Rica,

Costa Rica

mgach26@hotmail.com

\section{Ing. Tatiana Vargas Araya}

Ingeniera estructural, CARBON Ingeniería S.A., Costa Rica

tvargas@carboncr.com
Ing. Gabriela Arce Aita, M.Sc.

Ingeniera estructural, CARBON Ingeniería S.A., Costa Rica

garce@carboncr.com

Fecha de recepción: 21 SETIEMBRE 2017 / Fecha de aprobación: 15 DICIEMBRE 2017

Índices y Bases de Datos:

\section{lationdex ucRIndex}

\section{REDIB O Dialnet DOA}

revistas.ucr.ac.cr/index.php/materiales

(c) lanamme.ucr.ac.cr

@ metodosymateriales.lanamme@ucr.ac.cr
Políticas de Uso:

\section{(c) $($ i) $९$}

Revista Métodos y Materiales por LanammeUCR se distribuye bajo: Licencia Creative Commons Atribución-NoComercial-SinDerivar 4.0 Internacional. ISSN impreso: 2215-342X. ISSN electrónico: 2215-4558 


\section{Implementación de metodología para el diseño estructural de vigas-anillo} en tuberías a presión.

\section{THE USE OF A METHOD FOR STRUCTURAL DESIGN OF RING-GIRDERS USED IN PENSTOCKS.}

\section{Mónica Aguilera Chaves}

Estudiante Ingeniería Civil, Universidad de Costa Rica, Costa Rica

mgach26@hotmail.com

\section{Ing. Tatiana Vargas Araya}

Ingeniera estructural, CARBON Ingeniería S.A., Costa Rica

tvargas@carboncr.com
Ing. Gabriela Arce Aita, M.Sc.

Ingeniera estructural, CARBON Ingeniería S.A., Costa Rica

garce@carboncr.com

Fecha de recepción: 21 SETIEMBRE 2017 / Fecha de aprobación: 15 DICIEMBRE 2017

\section{RESUMEN}

Las fórmulas que estiman los esfuerzos en una tubería a presión restringida por una viga anillo fueron desarrolladas desde los años 30, sin embargo, su automatización no se ha implementado por los programas de cómputo comerciales debido a su alto grado de complejidad. Este trabajo consistió en automatizar la metodología para el cálculo de los esfuerzos internos generados en la sección transversal de una tubería de acero a presión expuesta sin juntas de expansión y restringida por una viga-anillo. Las cargas analizadas corresponden a las que se presentan en la condición normal de operación, este caso ocurre cuando la tubería forzada opera llena y a presión. La herramienta se elaboró empleando los lenguajes de programación de Mathcad 10 y EXCEL 2016 y permite calcular los esfuerzos internos que ocurren en los puntos de apoyo de las tuberías. El programa no toma en cuenta la carga de sismo.

PALABRAS CLAVE: Tubería a presión expuesta; acero; viga-anillo; combinaciones decargas; condición normal de operación; esfuerzos internos; programa.

\begin{abstract}
The methodology that calculates the internal stresses on a penstock shell restrained by a rigid ring girder was developed since the 1930's, however, its automatization has not been implemented by commercial use software due to its complexity. This projectintends to automate one methodology to calculate the internal stresses on a steel penstock shellrestrained bya ring-girder. A penstock under normal operation condition, this is apipe full of water under pressure, was analyzed. A calculating tool was produced using EXCEL 2016 and Mathcad 10, for calculating the internal stresseson the penstock's ring girder cross sections. The tool doesn't consider the earthquake loading conditions.

KEYWORDS: Exposed penstock; steel; ring-girder; load combinations; normal operation condition; internal stresses; spreadsheet.
\end{abstract}




\section{INTRODUCCIÓN}

Una de las aplicaciones de las tuberías a presión es aquella que las relaciona con la producción de electricidad en los proyectos hidroeléctricos. Estas cumplen con la función de transportar el agua desde el embalse o el tanque de oscilación hasta la casa de máquinas.

Las tuberías forzadas expuestas se colocan sobre dos tipos de apoyo: Tipo viga-anillo y tipo silla de montar, estos apoyos al restringir las deformaciones en el tubo generan esfuerzos localizados. En las Figuras 1 y 2 se puede observar el apoyo tipo viga-anillo.

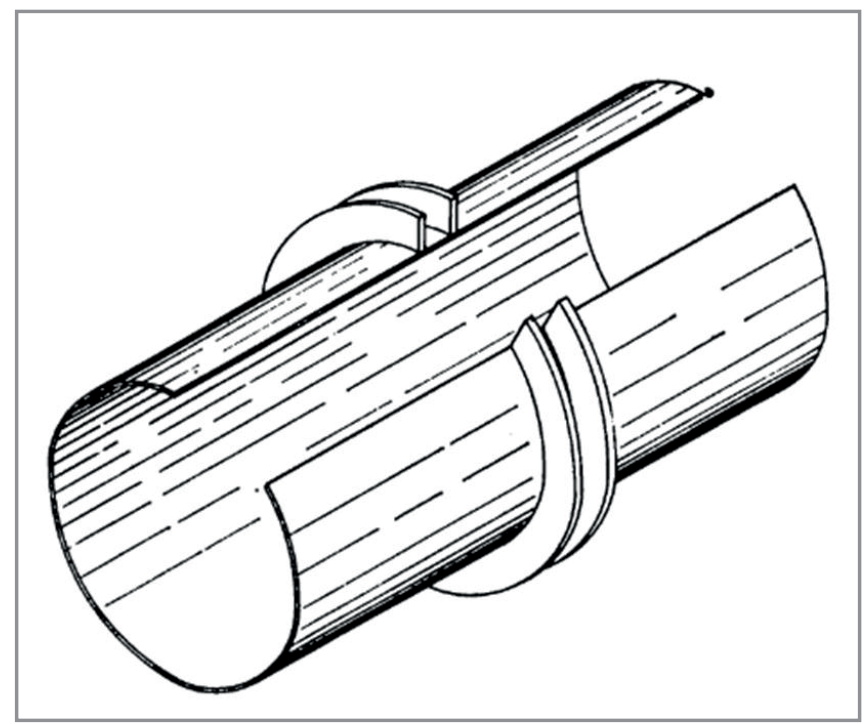

Figura 1. Viga-anillo soldada a la tubería (Garrett, 1948)

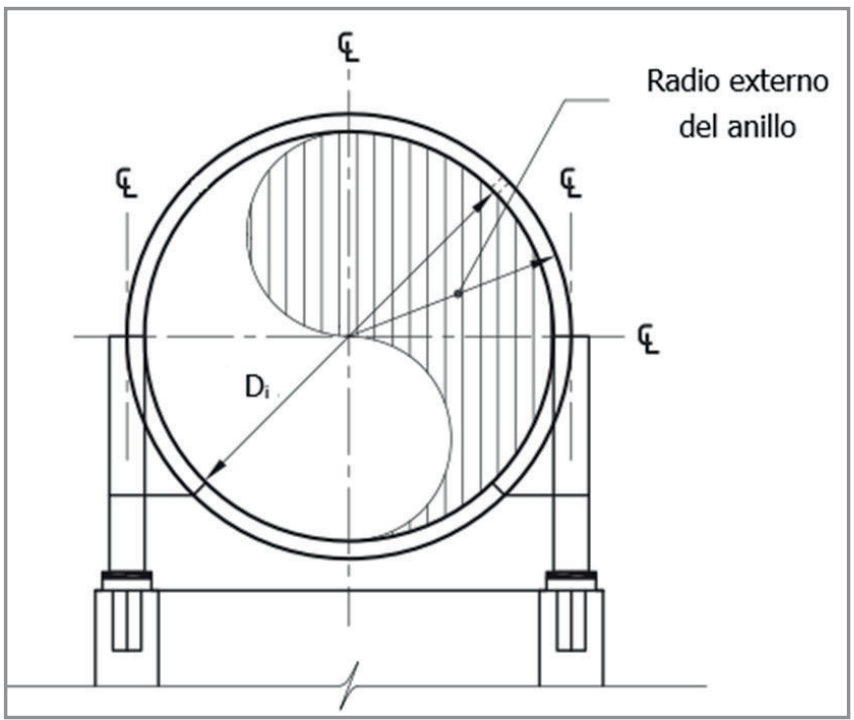

Figura 2. Vista frontal de la viga-anillo (ASCE, 2012)
La metodología existente para calcular los esfuerzos internos en las secciones de la tubería restringidas por vigas-anillo es engorrosa e iterativa, por esta razón en la práctica profesional es usual diseñar únicamente las estaciones más esforzadas. $\mathrm{Al}$ crear una herramienta de automatización se permite optimizar el diseño y por ende el costo final de la tubería.

Se realizó una revisión extensa de la bibliografía existente sobre el comportamiento y diseño estructural de tuberías a presión de acero sin juntas de expansión. Particularmente sobre la estimación de los esfuerzos internos en un tubo de pared delgada restringido por una viga-anillo sujeto a cargas de operación normal. Así como sobre los esfuerzos generados directamente en las secciones transversales de la viga-anillo.

\section{DESCRIPCIÓN DEL PROGRAMA}

El programa de automatización fue desarrollado combinando las plataformas de programación del Mathcad y el Visual Basic de EXCEL y se divide en tres fases. En la primera fase se recolectan los datos de entrada que describen la tubería y las presiones a las que se somete. En la segunda fase se calculan los esfuerzos internos en la sección transversal de la tubería restringida por la viga-anillo. Y en la tercera fase se generan los resultados en una interfase resumida de fácil lectura.

La metodología de diseño automatizada es la que se indica principalmente en Manuals and reports on engineering practice No. 79: steel penstocks (ASCE, 2012). Se parte de una tubería de presión sin juntas de expansión de acero, donde se quiere estudiar el comportamiento de un tramo de tubería comprendido entre dos cambios de dirección idealizados como apoyos tipo empotramiento, llamados PI en la Figura 3. El tramo de tubo se idealiza como una viga continua con apoyos simples ubicados en cada montura formada por una viga-anillo. La pieza de tubo con longitud $\mathrm{L}_{\mathrm{r}}$ (ver Figura 3) se ve afectada por la restricción a deformarse libremente producida la viga-anillo cuando la tubería se somete a presión. 


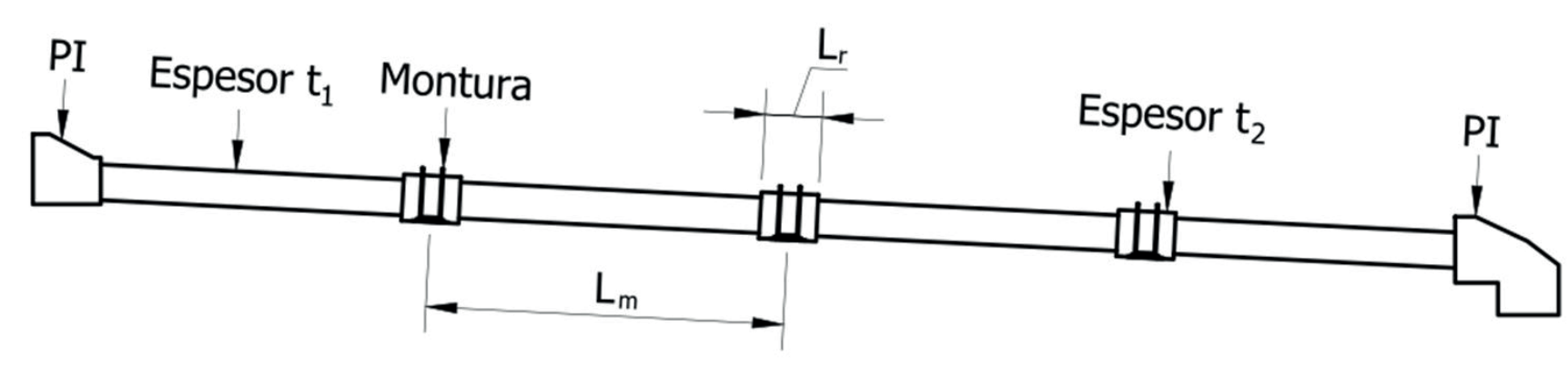

Figura 3. Tramo de tubería ubicado entre dos bloques de anclaje y con apoyos viga-anillo intermedios

A continuación, se detallan las cargas y los esfuerzos internos considerados por el programa.

\subsection{Cargas que actúan en la tubería a presión expuesta}

Las cargas que actúan en la tubería forzada bajo condición normal de operación son las siguientes:

- Peso de la estructura de acero

- Peso del agua contenida dentro de la tubería

- Presión interna para la condición de operación normal

- Acción de un cambio de temperatura que ocasiona expansión y contracción de la tubería.

Se excluyen de este estudio las cargas de viento y de sismo, las cuales también pueden ocurrir.

\subsection{Esfuerzos internos}

Las cargas que actúan en la tubería ocasionan esfuerzos en el sentido circunferencial y en el sentido longitudinal. Los esfuerzos en las tuberías forzadas se consideran biaxiales ya que poseen un espesor de pared delgado (Moss \& Basic, 2013).

\subsubsection{Esfuerzo longitudinal debido al efecto de Poisson}

El ASCE (ASCE, 2012) menciona que cuando la tubería se expande radialmente por la presión interna, ocurre una contracción en el sentido longitudinal debido al efecto de Poisson:

$$
\sigma_{L N}=\mu \frac{P_{N} r}{t_{2}^{\prime}}
$$

Donde $\sigma_{\mathrm{LN}}$ es el esfuerzo longitudinal por el efecto de Poisson $\left(\mathrm{kg} / \mathrm{cm}^{2}\right), \mathrm{P}_{\mathrm{N}}$ es la presión interna $\left(\mathrm{kg} / \mathrm{cm}^{2}\right), \mathrm{r}$ es el radio interno de la tubería $(\mathrm{cm}), \mathrm{t}_{2}$ es la resta entre el espesor de diseño $\left(t_{2}\right)$ y el espesor que se pierde por corrosión $(\mathrm{cm})$, y $\mu$ es la razón de Poisson, la cual es 0.3 para el acero.

\subsubsection{Esfuerzo longitudinal debido a los cambios de temperatura}

Los cambios de temperatura pueden ocasionar que la tubería sufra expansión o contracción. Estos esfuerzos se pueden estimar así (Chuan Peng \& Loong Peng, 2009):

$$
\sigma_{L \Delta T}=-\alpha E \Delta T
$$

$\sigma_{\mathrm{L} \Delta \mathrm{T}}$ es el esfuerzo longitudinal por el cambio de temperatura $\left(\mathrm{kg} / \mathrm{cm}^{2}\right), \alpha$ es el coeficiente de expansión del acero $(11.7 \mathrm{x}$ $\left.10^{-6} \mathrm{~m} / \mathrm{m} /{ }^{\circ} \mathrm{C}\right)$, E es el módulo de elasticidad del acero $(\mathrm{kg} /$ $\mathrm{cm}^{2}$ ) y $\Delta \mathrm{T}$ es el cambio de temperatura experimentado por la tubería $\left({ }^{\circ} \mathrm{C}\right)$.

\subsubsection{Esfuerzo longitudinal debido a la acción de la gravedad}

Un tramo de la tubería ubicado entre dos bloques de anclaje que se encuentre inclinado debe soportar la componente del peso que actúa en el sentido del eje (ASCE, 2012):

$$
\sigma_{L G}=\sum \frac{W_{t i} \sin \beta L_{\text {real } i}}{A_{2}}
$$

$\sigma_{\mathrm{LG}}$ es el esfuerzo longitudinal que ocurre por la componente axial del peso $\left(\mathrm{kg} / \mathrm{cm}^{2}\right), W_{t ~ i}$ es el peso del acero y del agua por unidad de longitud entre las monturas $(\mathrm{kg} / \mathrm{m})$ para un espesor de pared de tubería en específico $\left(t_{1 \mathrm{i}}\right), \beta$ es el ángulo de inclinación de la tubería (grados), $\mathrm{L}_{\text {real i }}$ es la longitud inclinada entre dos puntos de análisis adyacentes que tengan un espesor de tubería $\mathrm{t}_{1 \mathrm{i}}(\mathrm{m})$ y $\mathrm{A}_{2}$ es el área de la sección transversal de la tubería ubicada en el punto de apoyo $\left(\mathrm{cm}^{2}\right)$. 


\subsubsection{Esfuerzo longitudinal por la acción de viga}

Los tramos de la tubería actúan como vigas continuas que deben soportar su peso propio. Como las vigas-anillo previenen la distorsión de la sección transversal de la tubería en los puntos de apoyo (AISI, 1996), se puede usar la teoría simple de vigas para estimar estos esfuerzos:

$$
\sigma_{L B}=\frac{M r}{I_{2}}, M=\frac{W_{t} \cos \beta L_{m}^{2}}{9}
$$

$\sigma_{\mathrm{LB}}$ es el esfuerzo longitudinal por flexión $\left(\mathrm{kg} / \mathrm{cm}^{2}\right)$, M es el momento flector que ocurre en los apoyos $\left(\mathrm{kg}^{*} \mathrm{~m}\right), \mathrm{I}_{2}$ es la inercia de la sección transversal de la tubería en el punto de apoyo $\left(\mathrm{cm}^{4}\right)$ y $\mathrm{L}_{\mathrm{m}}$ es la distancia entre monturas $(\mathrm{m})$.

\subsubsection{Esfuerzo longitudinal por la acción restrictiva de la viga-anillo}

Debido al efecto restrictivo de la viga-anillo, la sección de la tubería no se puede expandir radialmente por la presión interna de la misma forma que lo hacen las secciones más lejanas al apoyo. Para satisfacer esa compatibilidad de deformaciones, se generan esfuerzos discontinuos. Estos esfuerzos se pueden determinar así (ASCE, 2012):

$$
\sigma_{L R N}=(1.82)\left(\frac{A_{r}-b t_{2}^{\prime}}{A_{r}+1.56 t_{2}^{\prime} \sqrt{r t_{2}^{\prime}}}\right)\left(\frac{P_{N} r}{t_{2}^{\prime}}\right)
$$

$\sigma_{\text {LRN }}$ es el esfuerzo longitudinal local que se produce por la restricción que le ocasiona la viga-anillo a la expansión radial de la tubería $\left(\mathrm{kg} / \mathrm{cm}^{2}\right)$, Ar es el área de la sección transversal de la viga-anillo que contempla también el área de la pared de la tubería que se encuentra confinada por el ancho b $\left(\mathrm{cm}^{2}\right)$, tal como se aprecia en la Figura 4.

En la Figura 5 se muestra el efecto restrictivo de la viga-anillo sobre la pared de la tubería.

\subsubsection{Esfuerzos longitudinales a compresión}

La combinación de los esfuerzos longitudinales que produzcan compresión, debe ser limitada para evitar que la tubería falle por pandeo (ASCE, 2012):

$$
\sigma_{\text {Lcr- }}=1.05 \times 10^{5} \frac{t_{2}}{r}
$$

Donde $\sigma_{\text {Lcr- }}$ es el esfuerzo crítico antes de que ocurra pandeo $\left(\mathrm{kg} / \mathrm{cm}^{2}\right)$.

\subsubsection{Esfuerzos cortantes por la acción de viga}

Los esfuerzos cortantes que se generan en la sección transversal de la tubería ocurren por el peso del acero y del agua (ASCE, 2012). Actúan tangentes a todos los puntos de la sección transversal:

$$
\tau_{B}=\frac{2 Q_{t} \cos \beta \sin \theta}{\pi D_{m} t_{2}^{\prime}}
$$
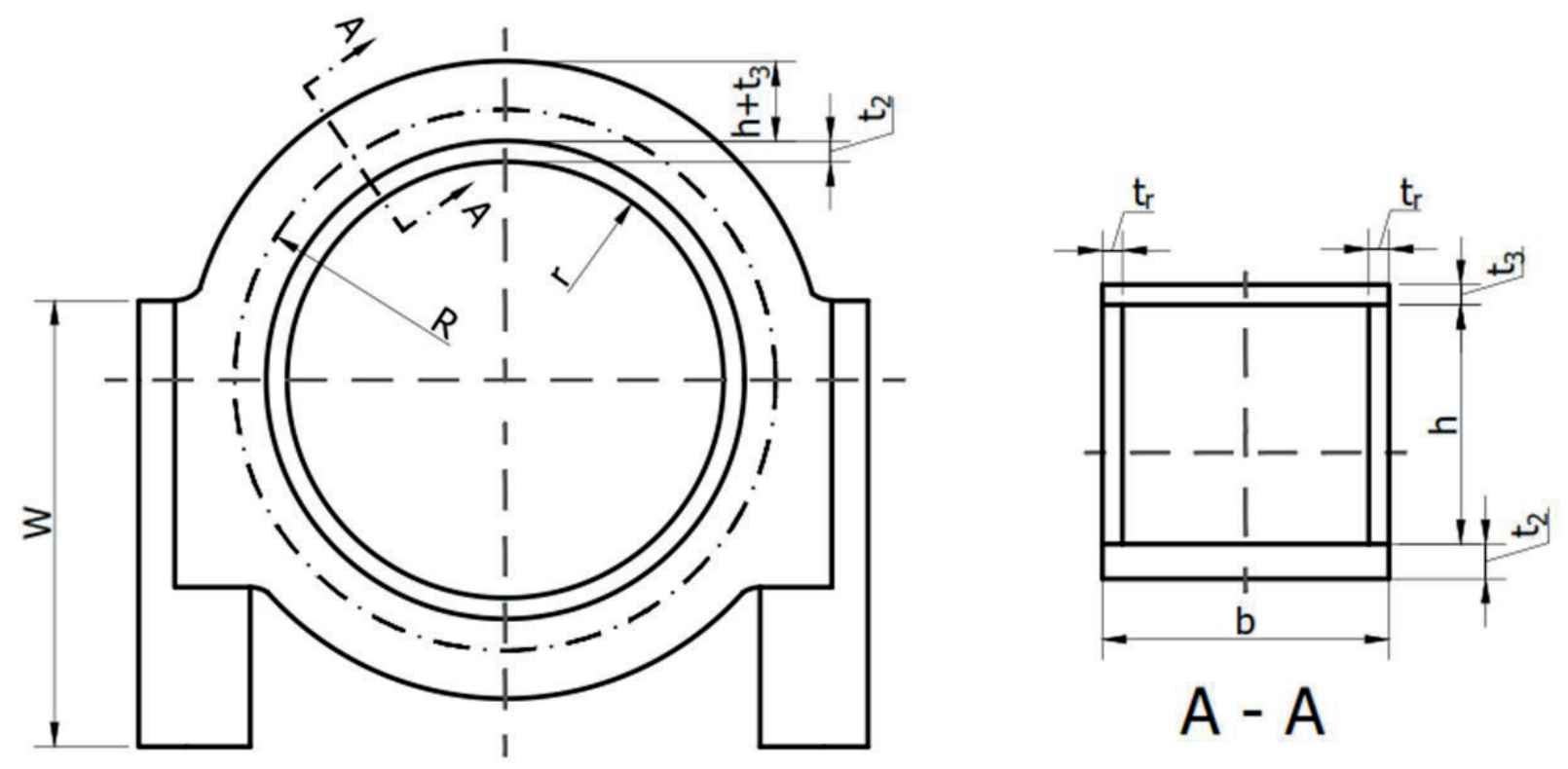

Figura 4. Área de la viga-anillo y de la pared de la tubería confinada dentro del ancho b 


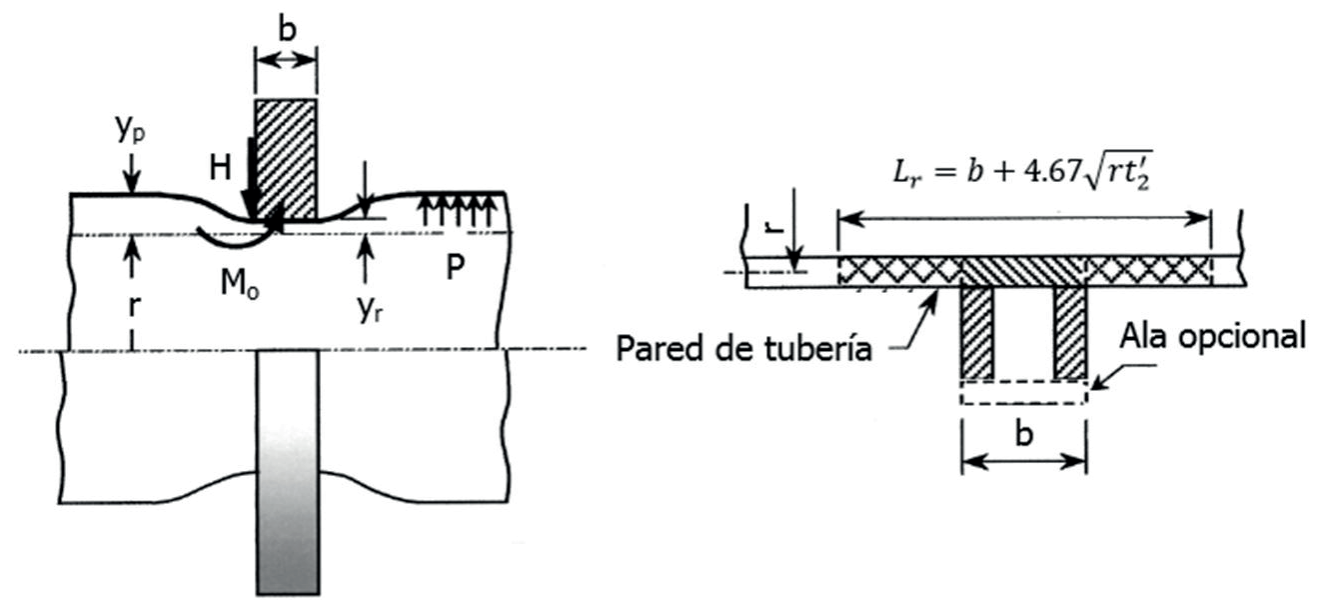

Figura 5. Efecto de la restricción del anillo en la tubería (Chuan Peng \& Loong Peng, 2009)

$\tau_{B}$ es el esfuerzo cortante que se genera en la sección $(\mathrm{kg} /$ $\left.\mathrm{cm}^{2}\right), Q_{t}$ es el peso del acero y del agua en una luz ( $\left.\mathrm{kg}\right), \theta$ es el ángulo con respecto al cenit del anillo donde se calcula el esfuerzo (grados), $\mathrm{D}_{\mathrm{m}}$ es el diámetro medio de la tubería $(\mathrm{cm})$.

\subsubsection{Esfuerzos circunferenciales por el efecto de la presión interna}

En la sección transversal de la viga-anillo actúan fuerzas circunferenciales producidas por la presión interna. Se pueden calcular de la siguiente manera (USBR, 1986):

$$
N_{r N}=P_{N} r\left[b+\frac{2\left(1-\mu^{2}\right)}{q}\right], q=\frac{1.285}{\sqrt{r t_{2}^{\prime}}}
$$

$\mathrm{N}_{\mathrm{rN}}$ es la fuerza circunferencial de tensión que se produce por la presión interna $(\mathrm{kg})$ y q es el factor característico de la tubería $\left(\mathrm{cm}^{-1}\right)$.

\subsubsection{Esfuerzos circunferenciales por las cargas gravitatorias}

El peso de la tubería y del agua se transmite a la viga-anillo mediante fuerzas cortantes tangenciales que se producen en la junta entre la tubería y la viga-anillo (Troitsky, 1990). Dichas fuerzas cortantes ocasionan fuerzas internas en la viga-anillo (un momento interno, una fuerza axial y una fuerza cortante). Adicionalmente, la viga-anillo debe soportar las reacciones que provienen de las columnas de soporte (ver Figura 4), las cuales pueden ser excéntricas con respecto al centroide de la viga-anillo, por lo que se generan momentos flectores que actúan en el ecuador del anillo. Para estimar las fuerzas internas por esas cargas, el USBR (USBR, 1986) publicó una tabla con coeficientes:

\begin{tabular}{|c|c|c|c|c|c|c|}
\hline \multicolumn{6}{|c|}{ Tabla 1. Coeficientes para estimar las fuerzas internas en la viga-anillo (USBR, 1986) } \\
\hline \multirow{2}{*}{$\theta$} & \multicolumn{2}{|c}{ Dr } & \multicolumn{2}{c|}{ Mr } & \multicolumn{2}{c|}{ Vr } \\
\cline { 2 - 7 } & K1 & K2 & K3 & K4 & K5 & K6 \\
\hline $0^{\circ}$ & -0.238732 & 0.31831 & 0.011267 & -0.06831 & 0 & 0 \\
\hline $15^{\circ}$ & -0.241384 & 0.307464 & 0.008618 & -0.057464 & 0.019651 & 0.082385 \\
\hline $30^{\circ}$ & -0.248415 & 0.275664 & 0.001585 & -0.025665 & 0.0328 & 0.159155 \\
\hline $45^{\circ}$ & -0.257198 & 0.225079 & -0.007198 & 0.024921 & 0.032117 & 0.225079 \\
\hline $60^{\circ}$ & -0.263704 & 0.159155 & -0.013704 & 0.090845 & 0.014417 & 0.275664 \\
\hline $75^{\circ}$ & -0.263023 & 0.082385 & -0.013023 & 0.167616 & -0.022945 & 0.307463 \\
\hline $89.9^{\circ}$ & -0.25 & 0 & 0 & 0.25 & -0.079577 & 0.31831 \\
\hline$-89.9^{\circ}$ & 0.25 & 0 & 0 & -0.25 & -0.079577 & 0.31831 \\
\hline $105^{\circ}$ & 0.263023 & -0.082385 & 0.013023 & -0.167616 & -0.022945 & 0.307463 \\
\hline $120^{\circ}$ & 0.263704 & -0.159155 & 0.013704 & -0.090845 & 0.014417 & 0.275664 \\
\hline $135^{\circ}$ & 0.257198 & -0.225079 & 0.007198 & -0.024921 & 0.032117 & 0.225079 \\
\hline $150^{\circ}$ & 0.248415 & -0.275664 & -0.001585 & 0.025665 & 0.0328 & 0.159155 \\
\hline $165^{\circ}$ & 0.241384 & -0.307464 & -0.008618 & 0.057464 & 0.019651 & 0.082385 \\
\hline $180^{\circ}$ & 0.238732 & -0.31831 & -0.011267 & 0.06831 & 0 & 0 \\
\hline
\end{tabular}


Las fuerzas internas que se generan en la sección transversal de la viga-anillo se representan en la Figura 6.

Sistema de fuerzas externo
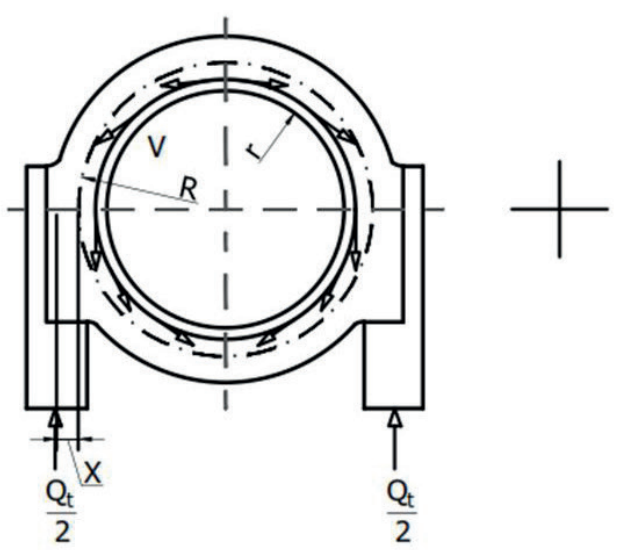

Sistema de fuerzas interno
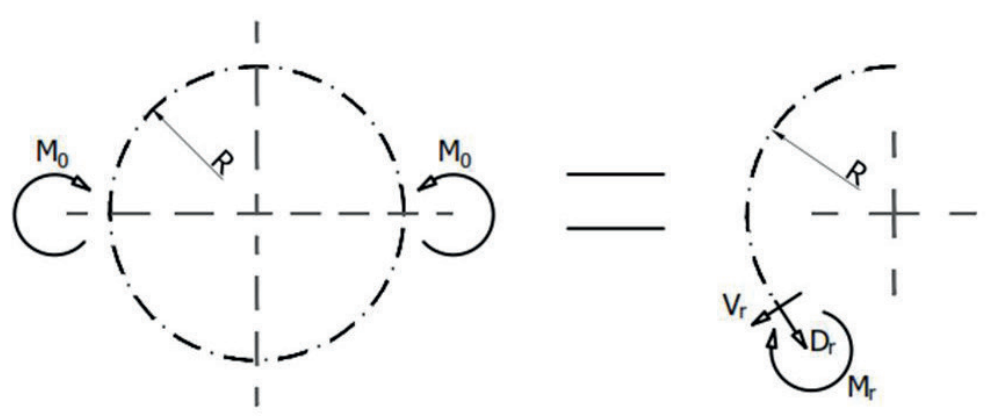

Figura 6. Fuerzas gravitatorias actuantes en la viga-anillo

El momento interno, $\mathrm{Mr}\left(\mathrm{kg}^{*} \mathrm{~cm}\right)$, la fuerza axial, $\mathrm{Dr}(\mathrm{kg})$, y la fuerza cortante radial, $\operatorname{Vr}(\mathrm{kg})$, se calculan así:

$$
\begin{gathered}
D_{r}=Q_{t}\left(K_{1}+B K_{2}\right) \\
M_{r}=Q_{t}\left(R K_{3}+X K_{4}\right) \\
V_{r}=Q_{t}\left(K_{5}+C K_{6}\right)
\end{gathered}
$$

B y C se calculan como se muestra a continuación:

$$
\begin{gathered}
K=\frac{r}{L_{m}}\left[\frac{\mu L_{m}^{2}}{12 r^{2}}+\left(1-\mu^{2}\right)\left(1-\frac{Q_{s 1}}{2 Q_{t}}\right)+\frac{(2+\mu) L_{m}}{4 q r^{2}}\right] \\
B=\frac{r}{R}\left(1-\frac{2 K}{q r}\right)-\frac{X}{R} \\
C=\left(\frac{r}{R}-1\right)\left(1-\frac{2 K}{q r}\right)-\frac{X}{R}
\end{gathered}
$$

Donde $\mathrm{Q}_{\mathrm{s} 1}$ es el peso de la tubería de acero vacía en una luz $(\mathrm{kg}), \mathrm{R}$ es el radio al centroide de la sección compuesta de la viga-anillo con la tubería $(\mathrm{cm})$ y $\mathrm{X}$ es la excentricidad que existe entre el centroide de dicha sección compuesta y el centroide de las columnas de soporte de las monturas $(\mathrm{cm})$.

\subsubsection{Esfuerzos circunferenciales totales}

Mediante el principio de superposición se pueden estimar los esfuerzos totales que ocurren en la fibra interior y en la fibra exterior de la viga-anillo (USBR, 1986):

$$
\begin{aligned}
\sigma_{\text {CTNSup }} & =\frac{D_{r}}{A_{C}}-M_{r} \frac{\bar{x}}{I_{c}}+\frac{N_{r N}}{A_{c}} \\
\sigma_{\text {CTNinf }} & =\frac{D_{r}}{A_{c}}+M_{r} \frac{\bar{Z}}{I_{c}}+\frac{N_{r N}}{A_{c}}
\end{aligned}
$$

El área y la inercia $\left(A_{c}\right.$ e $\left.I_{c}\right)$ se toman a partir de una sección transversal que combina la viga-anillo en conjunto con un ancho efectivo $\left(\mathrm{L}_{\mathrm{e}}\right)$ de la pared de la tubería (AWWA, 2004):

$$
L_{e}=1.56 \sqrt{r t_{2}^{\prime}}+b
$$

$\bar{x}$ es la posición del centroide desde la fibra superior de la sección compuesta $(\mathrm{cm}), \bar{z}$ es la posición del centroide desde la fibra inferior de la sección combinada $(\mathrm{cm}), \sigma_{\text {CTNinf }}$ y $\sigma_{\text {CTNinf }}$ corresponden al esfuerzo circunferencial total en la sección combinada en la fibra inferior y en la fibra superior de la sección combinada $\left(\mathrm{kg} / \mathrm{cm}^{2}\right)$, respectivamente.

\subsubsection{Esfuerzos cortantes que actúan en el alma de la viga-anillo}

Por las fuerzas cortantes radiales, $\mathrm{V}_{\mathrm{r}}$, se generan esfuerzos cortantes en el alma de la viga-anillo. Estos se pueden estimar con la teoría simple de vigas (Chang, 1980):

$$
\tau_{r}=\frac{V_{r} Q_{r}}{2 I_{c} t_{r}}
$$

Donde $\mathrm{Q}_{\mathrm{r}}$ es el momento estático respecto al eje neutro del área parcial de la sección transversal situada a un lado del corte imaginario en la viga-anillo $\left(\mathrm{cm}^{3}\right)$. 


\subsubsection{Esfuerzos equivalentes}

Los esfuerzos totales longitudinales, los esfuerzos totales circunferenciales y los esfuerzos cortantes debido al efecto de viga pueden ser combinados en la fibra interior de la viga-anillo a cada 15 grados de la circunferencia. Para ello se puede emplear la teoría de la energía de la máxima distorsión (Hydraulic Gate and Penstock Association, 1981):

$$
\sigma_{e q}=\sqrt{\sigma_{C T N i n f}^{2}-\sigma_{C T N i n f} \sigma_{L T N}+\sigma_{L T N}^{2}+3 \tau_{B}^{2}}
$$

Donde $\sigma_{\text {eq }}$ es el esfuerzo equivalente que se produce en un punto de la circunferencia en la fibra interna de la sección transversal de la tubería $\left(\mathrm{kg} / \mathrm{cm}^{2}\right), \sigma_{\text {LTN }}$ es el esfuerzo longitudinal total que actúa en la fibra interna de un punto de la sección transversal de la tubería $\left(\mathrm{kg} / \mathrm{cm}^{2}\right)$.

\subsubsection{Esfuerzos admisibles}

Se debe verificar que las solicitaciones sean menores que los esfuerzos admisibles. Para esto, los esfuerzos totales circunferenciales, longitudinales y los combinados se clasifican dependiendo del tipo de carga y del riesgo que representen para la estructura. La clasificación es la siguiente (ASCE, 2012):

- Esfuerzos primarios (P): Cuando estos esfuerzos exceden la fluencia del material, pueden ocasionar la falla de la estructura o grandes deformaciones. Dentro de esta clasificación se encuentran los esfuerzos producidos por la presión interna y por las cargas gravitatorias.

- Esfuerzos secundarios (Q): Estos esfuerzos se producen por restricciones al movimiento de la tubería. Tal es el caso de los esfuerzos que se generan por los cambios de temperatura y por el efecto restrictivo de la viga-anillo.

La revisión parte del esfuerzo admisible propio del material del tubo que se denomina esfuerzo admisible básico y es el menor entre el esfuerzo de fluencia mínimo del acero entre 1.5 y el esfuerzo último entre 2.4 (ASCE, 2012). Luego, dependiendo de la clasificación de la combinación de esfuerzos que se está comparando, el esfuerzo admisible básico se ajusta con coeficientes para permitir mayores magnitudes.

Antes de que los esfuerzos circunferenciales y longitudinales sean comparados con los admisibles, se ajustan con la eficiencia de junta. Esta variable es 1 para esfuerzos a compresión, pero para esfuerzos a tensión toma valores entre 0.5 y 1 dependiendo del tipo de soldadura que se le realice a la tubería y del grado de inspección que reciban (ASCE, 2012).

\section{COMPOSICIÓN DEL PROGRAMA}

Tal y como se indicó anteriormente, el programa se encuentra compuesto por tres etapas:

- Ingreso de datos de entrada en lenguaje de programación de Visual Basic

- Cálculo de los esfuerzos internos en Mathcad 10

- Presentación de resultados en Visual Basic

En la Figura 7 se muestra la interfaz del ingreso de datos de entrada del programa y en la Figura 8 se pueden observar parte de los resultados del programa para un caso específico. No se incluye una figura de la segunda etapa porque es una extensa hoja de cálculo en Mathcad 10, sin embargo, el lector puede acceder a más información de este programa dirigiéndose a una tesis de grado de licenciatura (Aguilera, 2017).

Los datos de entrada son exportados al Mathcad 10 y de ahí se exportan al otro EXCEL que tiene como objetivo presentar los resultados de manera más compacta para que le permitan al usuario una fácil inspección. Por ejemplo, para una montura en particular, se muestran los resultados de los esfuerzos a cada $15^{\circ}$ de la circunferencia de la tubería, esto se aprecia en la Figura 8. 


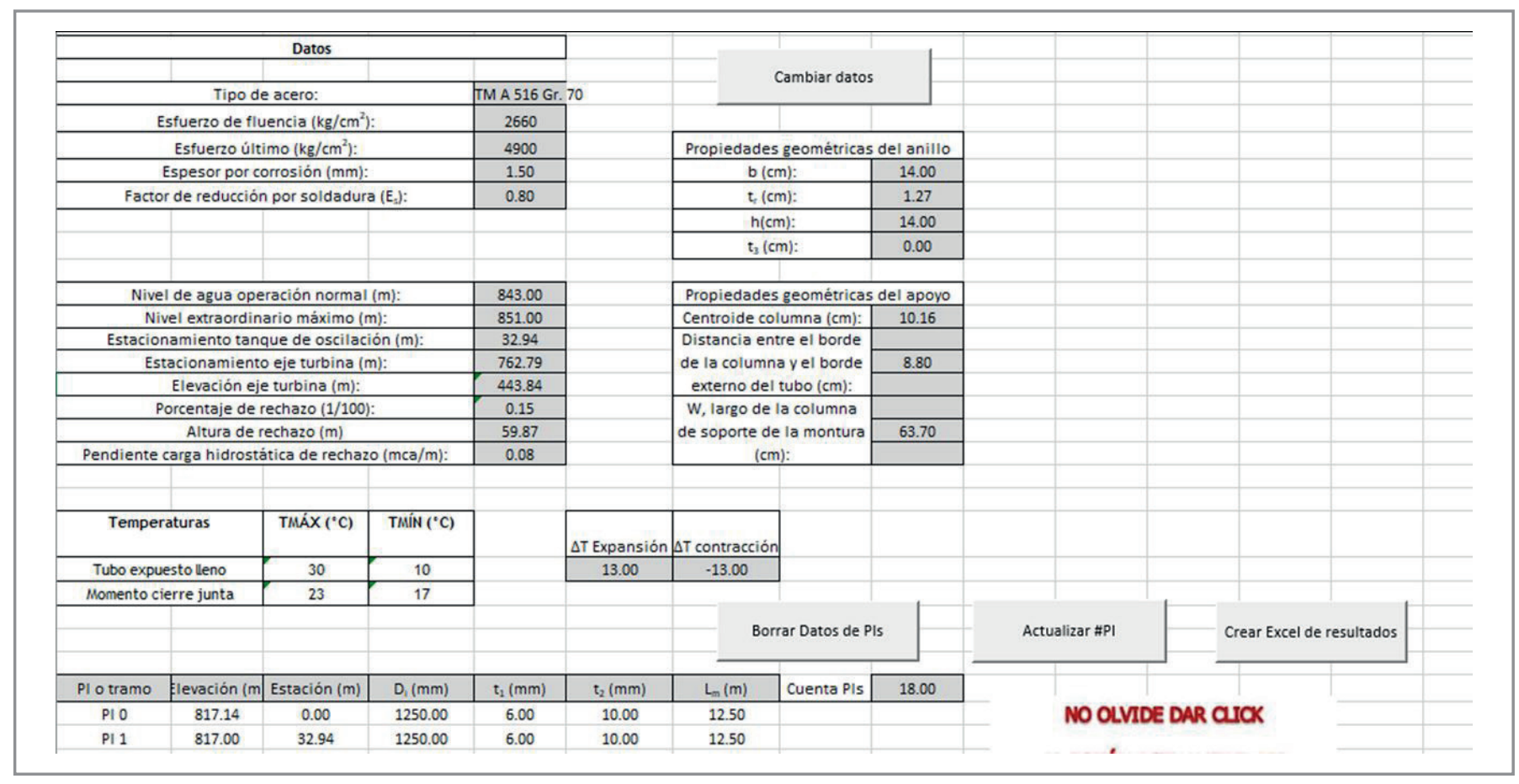

Figura 7. Interfaz de ingreso de datos de entrada al programa

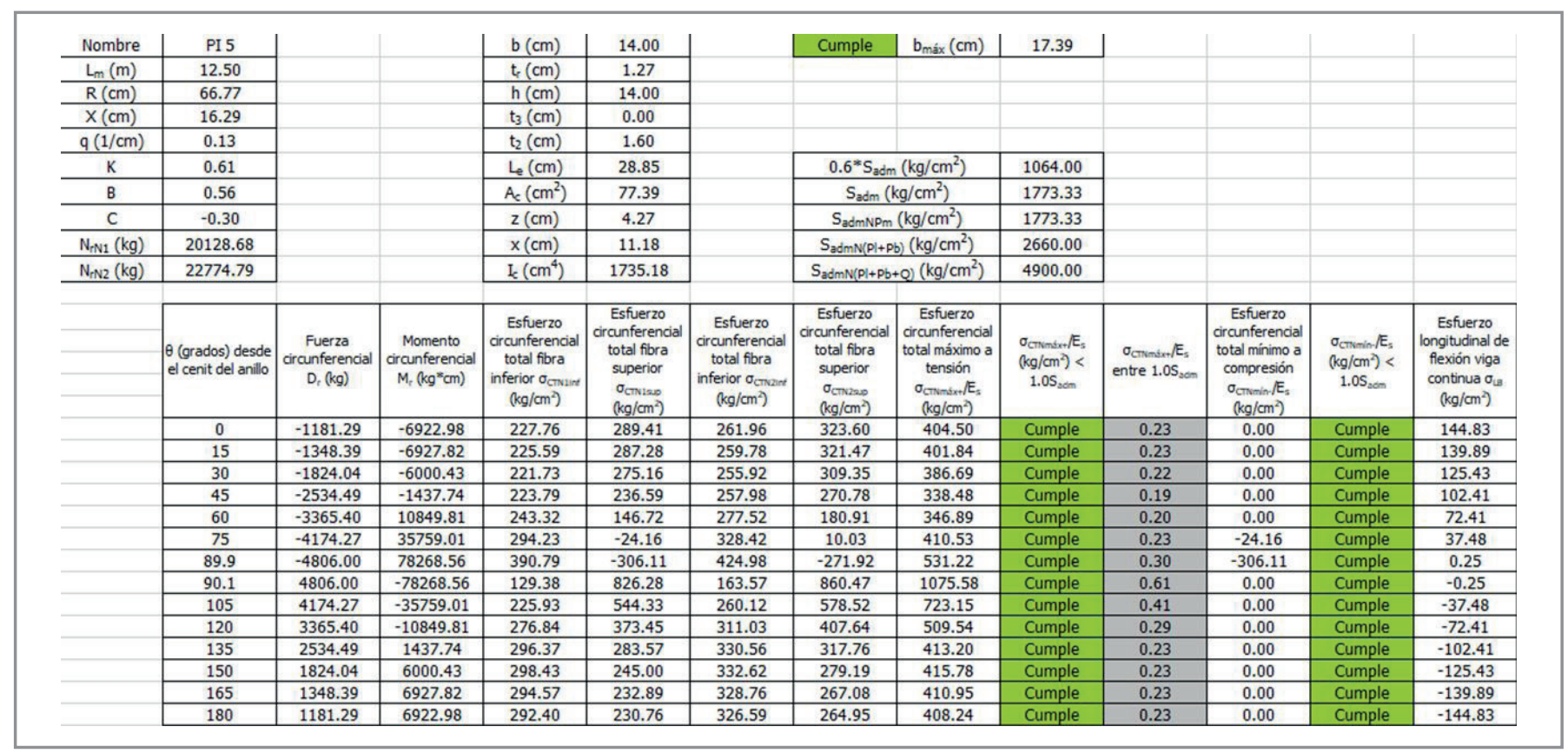

Figura 8. Presentación de los resultados del programa para una montura en específico 


\section{COMPARACIÓN DE RESULTADOS}

Se realizó el ejercicio de comparar los resultados arrojados por el programa con los resultados obtenidos de diseños realizados por la empresa CARBON Ingeniería S.A. En la Tabla 2 se muestran las características estructurales de una tubería:

\begin{tabular}{|c|c|}
\hline $\begin{array}{l}\text { Tabla 2. Características estruct } \\
\text { presión (CARBON Ingen }\end{array}$ & $\begin{array}{l}\text { s de una tubería a } \\
\text { S.A., 2006) }\end{array}$ \\
\hline Acero & ASTM A 516 Gr. 70 \\
\hline Espesor de corrosión $t_{c}(\mathrm{~mm})$ & 1.5 \\
\hline Eficiencia de junta $\mathrm{E}_{\mathrm{s}}$ & 0.8 \\
\hline Cambio de temperatura $\Delta \mathrm{T}_{\text {máx }}\left({ }^{\circ} \mathrm{C}\right)$ & 13 \\
\hline Cambio de temperatura $\Delta \mathrm{T}_{\min }\left({ }^{\circ} \mathrm{C}\right)$ & -13 \\
\hline Diámetro interno $D_{i}(\mathrm{~mm})$ & 1250 \\
\hline Separación entre monturas $\mathrm{L}_{\mathrm{m}}(\mathrm{m})$ & 12.5 \\
\hline
\end{tabular}

En la Tabla 3 se muestran los datos hidráulicos necesarios para diseñar la tubería. Allí se presenta una variable que se denomina porcentaje de rechazo, el cual se emplea para estimar las sobrepresiones que se generan producto del golpe de ariete.

Tabla 3. Datos hidráulicos necesarios para el diseño de la tubería forzada en análisis (CARBON Ingeniería S.A., 2006)

\begin{tabular}{|c|c|}
\hline Nivel de aguas de operación normal $(\mathrm{m}):$ & 843 \\
\hline Nivel extraordinario máximo $(\mathrm{m}):$ & 851 \\
\hline Estacionamiento del tanque de oscilación $(\mathrm{m}):$ & 32.944 \\
\hline Estacionamiento del eje de la turbina $(\mathrm{m}):$ & 762.793 \\
\hline Elevación del eje de la turbina $(\mathrm{m}):$ & 443.840 \\
\hline Porcentaje de rechazo $(1 / 100):$ & 0.15 \\
\hline
\end{tabular}

En la Tabla 4 se presentan las dimensiones de la viga-anillo y de la columna de soporte:

\begin{tabular}{|c|c|}
\hline Ancho del alma b $(\mathrm{cm})$ & 14 \\
\hline Espesor de las placas del alma $t_{r}(\mathrm{~cm})$ & 1.27 \\
\hline Altura del alma $\mathrm{h}(\mathrm{cm})$ & 14 \\
\hline Centroide de la columna de soporte $\mathrm{c}(\mathrm{cm})$ & 10.16 \\
\hline $\begin{array}{c}\text { Distancia entre la pared de la columna } \\
\text { y la pared de la tubería } \mathrm{d}(\mathrm{cm})\end{array}$ & 8.8 \\
\hline
\end{tabular}

Se eligieron tres tramos para efectuar el ejercicio de calibración: PI 4 al PI 5, PI 8 al PI 9 y PI 9 al PI 10. En la Tabla 5 se muestran las coordenadas de los puntos de mayor interés, que consisten en bloques de anclaje (PI) y cambios de espesor (CT).

Tabla 5. Coordenadas de los PI empleados para verificar el programa (CARBON. Ingeniería S.A., 2006)

$\mathrm{PI}$ o punto de cambio de espesor Elevación $(\mathrm{m}) \quad$ Estación $(\mathrm{m})$

\begin{tabular}{|c|c|c|}
\hline PI 4 & 727.377 & 178.255 \\
\hline CT 4 1 & - & 219.727 \\
\hline PI 5 & 725.816 & 220.727 \\
\hline PI 8 & 656.47 & 395.545 \\
\hline CT 8 1 & - & 472.802 \\
\hline PI 9 & 621.498 & 473.715 \\
\hline CT 9 1 & - & 549.019 \\
\hline PI 10 & 500.071 & 640.343 \\
\hline
\end{tabular}

La primera parte del ejercicio radicó en calcular de forma independiente (sin uso de la herramienta) los esfuerzos a cada $15^{\circ}$ de algunas secciones transversales de la tubería donde se ubicaban las monturas y en compararlas con los resultados del programa. En el primer tramo, se calcularon los esfuerzos para una montura idealizada ubicada en el punto de cambio de espesor CT 41 , específicamente a $75^{\circ}$ y a $180^{\circ}$. Del segundo tramo se tomó una montura ubicada de manera idealizada en el cambio de espesor CT 81 y se calcularon los esfuerzos a $30^{\circ}$. Y en el último tramo se tomaron dos monturas reales y se calcularon los esfuerzos a $90^{\circ}$ y a $165^{\circ}$, respectivamente. Como los cálculos son extensos, no se estimaron los esfuerzos en todos los puntos de las secciones transversales, pero se tuvo el cuidado de no repetir los ángulos para así concluir que el código del programa estuviera funcionando correctamente.

Para la segunda parte del ejercicio, se compararon los resultados del programa con la memoria de cálculo de la empresa CARBON Ingeniería S.A.. En este caso se tomaron los puntos PI 5, PI 9 y PI 10 para efectuar la equiparación, debido a que, para cada uno de esos tramos (PI 4 al PI 5, PI 8 al PI 9 y PI 9 al PI 10), el espesor de la tubería en la cercanía de la montura se escogía analizando el punto de mayor presión interna. Acá (ver Tablas 6, 7 y 8) se muestran resumidas las diferencias encontradas en los esfuerzos internos. 
Tabla 6. Cálculos en la sección transversal restringida

por la viga-anillo $\mathrm{PI} 5, \theta=180^{\circ}$

Variable CARBON Ingeniería S.A. Programa Diferencia

\begin{tabular}{|c|c|c|c|}
\hline$\sigma_{\mathrm{LG}}\left(\mathrm{kg} / \mathrm{cm}^{2}\right)$ & -4.168 & -4.002 & $3.97 \%$ \\
\hline$\sigma_{\mathrm{LB}}\left(\mathrm{kg} / \mathrm{cm}^{2}\right)$ & -149.948 & -144.829 & $3.41 \%$ \\
\hline$\sigma_{\text {LTN1C }}\left(\mathrm{kg} / \mathrm{cm}^{2}\right)$ & 739.21 & 744.494 & $0.72 \%$ \\
\hline$\sigma_{\text {LTN2C }}\left(\mathrm{kg} / \mathrm{cm}^{2}\right)$ & 814.657 & 819.941 & $0.65 \%$ \\
\hline$\tau_{\mathrm{B}}\left(\mathrm{kg} / \mathrm{cm}^{2}\right)$ & - & 0 & - \\
\hline$\sigma_{\text {eqN } 1 \mathrm{C}}\left(\mathrm{kg} / \mathrm{cm}^{2}\right)$ & 644.814 & 812.096 & $25.94 \%$ \\
\hline$\sigma_{\text {eqN2C }}\left(\mathrm{kg} / \mathrm{cm}^{2}\right)$ & 710.119 & 893.711 & $25.85 \%$ \\
\hline
\end{tabular}

Tabla 7. Cálculos en la sección transversal restringida por la viga-anillo $\mathrm{PI} 9, \theta=30^{\circ}$

Variable CARBON Ingeniería S.A. Programa Diferencia

\begin{tabular}{|c|c|c|c|}
\hline$\sigma_{\text {LG }}\left(\mathrm{kg} / \mathrm{cm}^{2}\right)$ & -73.802 & -68.476 & $7.22 \%$ \\
\hline$\sigma_{\text {LB }}\left(\mathrm{kg} / \mathrm{cm}^{2}\right)$ & 0 & 89.69 & - \\
\hline$\sigma_{\text {LTN1C }}\left(\mathrm{kg} / \mathrm{cm}^{2}\right)$ & 883.261 & 978.276 & $10.76 \%$ \\
\hline$\sigma_{\text {LTN2C }}\left(\mathrm{kg} / \mathrm{cm}^{2}\right)$ & 987.355 & 1082.37 & $9.62 \%$ \\
\hline$\tau_{\mathrm{B}}\left(\mathrm{kg} / \mathrm{cm}^{2}\right)$ & - & 24.079 & - \\
\hline$\sigma_{\text {eqN1C }}\left(\mathrm{kg} / \mathrm{cm}^{2}\right)$ & 767.609 & 1068.981 & $39.26 \%$ \\
\hline$\sigma_{\text {eqN2c }}\left(\mathrm{kg} / \mathrm{cm}^{2}\right)$ & 856.454 & 1178.581 & $37.61 \%$ \\
\hline
\end{tabular}

Tabla 8. Cálculos en la sección transversal restringida por la viga-anillo PI $10, \theta=165^{\circ}$

\begin{tabular}{|c|c|c|c|}
\hline Variable & CARBON Ingeniería S.A. & Programa & Diferencia \\
\hline$\sigma_{\mathrm{LG}}\left(\mathrm{kg} / \mathrm{cm}^{2}\right)$ & -265.647 & -261.399 & $1.60 \%$ \\
\hline$\sigma_{\mathrm{LB}}\left(\mathrm{kg} / \mathrm{cm}^{2}\right)$ & 0 & -91.817 & - \\
\hline $\begin{array}{c}\sigma_{\text {LTN1C }}(\mathrm{kg} / \\
\left.\mathrm{Cm}^{2}\right)\end{array}$ & 1040.976 & 953.407 & $8.41 \%$ \\
\hline $\begin{array}{c}\sigma_{\text {LTN2c }}(\mathrm{kg} / \\
\left.\mathrm{Cm}^{2}\right)\end{array}$ & 1184.421 & 1096.852 & $7.39 \%$ \\
\hline$\tau_{\mathrm{B}}\left(\mathrm{kg} / \mathrm{cm}^{2}\right)$ & - & 11.44 & - \\
\hline $\begin{array}{c}\sigma_{\text {eqN1C }}(\mathrm{kg} / \\
\left.\mathrm{Cm}^{2}\right)\end{array}$ & 914.934 & 1062.103 & $16.09 \%$ \\
\hline $\begin{array}{c}\sigma_{\text {eqN2C }}(\mathrm{kg} / \\
\left.\mathrm{cm}^{2}\right)\end{array}$ & 1040.987 & 1219.336 & $17.13 \%$ \\
\hline \multicolumn{2}{|c|}{}
\end{tabular}

\section{DISCUSIÓN DE RESULTADOS}

Los cálculos realizados independientemente coincidieron con las salidas del programa, con esto se verificó que el código funciona adecuadamente.

Se encontraron varias diferencias en la comparación de resultados con la memoria de cálculo de CARBON Ingeniería S.A.. Estas se dan porque el programa elaborado realiza un análisis más exhaustivo de los esfuerzos. En el caso del esfuerzo por efecto de la gravedad, $\sigma_{L G}$, las diferencias se presentan porque el programa considera en su código la posibilidad de que existan cambios de espesor en los tramos y calcula el esfuerzo de manera más precisa. Con respecto al esfuerzo por el efecto de viga, $\sigma_{\mathrm{LB}}$, las diferencias ocurren porque el programa calcula ese esfuerzo para cada $15^{\circ}$ de la circunferencia de la tubería, en cambio en la memoria de CARBON Ingeniería se calculan para las fibras extremas $\left(0^{\circ}\right.$ y $180^{\circ}$, exclusivamente). Las diferencias anteriores se arrastran a los esfuerzos longitudinales totales $\sigma_{\text {LTN1c }}$ y $\sigma_{\text {LTN2c }}$. Adicionalmente, con esta herramienta se pueden calcular los esfuerzos debido al cortante de viga, $\tau_{B}$, los cuales fueron despreciados en la memoria de cálculo de CARBON Ingeniería S.A. por su poca magnitud. Por otro lado, los esfuerzos equivalentes $\sigma_{\text {eqN1c }}$ y $\sigma_{\text {eq N2c }}$ fueron los que exhibieron mayores diferencias, ya que la metodología contemplada por el programa sigue las recomendaciones del ASCE en cuanto a ajustar las solicitaciones con la eficiencia de junta antes de combinar los esfuerzos y además, se toma en cuenta el esfuerzo cortante de viga en la ecuación de la energía de máxima distorsión.

\section{CONCLUSIONES}

La metodología existente para calcular los esfuerzos internos en las secciones transversales de la tubería restringidas por vigas-anillo ha experimentado pequeñas variaciones desde que se promovió el uso de este tipo de apoyo en tuberías forzadas. Esta metodología es engorrosa e iterativa lo cual implica invertir muchas horas en el cálculo manual iterativo para cada una de las monturas de una tubería. Por lo tanto, el uso del programa minimiza los posibles errores asociados a cálculos manuales y permite la repetición de su uso en poco tiempo.

Además, se lograron comparar los resultados del programa con cálculos independientes de una tubería forzada diseñada por CARBON Ingeniería S.A.. Y se determinó que las variaciones en los esfuerzos rondan un máximo del 10\%, a excepción de los esfuerzos equivalentes donde las diferencias son del $30 \%$ por el ajuste con la eficiencia de junta. 
El programa permite realizar análisis más exhaustivos, debido a la posibilidad de analizar los esfuerzos en las secciones transversales ubicadas en monturas intermedias entre bloques de anclaje.

\section{REFERENCIAS BIBLIOGRÁFICAS}

- Aguilera, M. (2017). Automatización de una metodología para el diseño estructural de monturas tipo viga-anillo en tuberías a presión expuestas de acero. (Tesis de grado). Universidad de Costa Rica.

American Iron and Steel Institute (AISI). (1996). Welded steel pipe, steel plate engineering data, Vol. 3. Washington, D.C.

- American Society of Civil Engineers (ASCE). (2012). Manuals and reports on engineering practice No. 79: steel penstocks (2da ed. en inglés). Virginia: ASCE.

- American Water Works Association (AWWA). (2004). M11 Manual: A steel penstocks guide for design and installation (4ta ed. en inglés). Dénver: AWWA.

- Chang, D. (1980). Ring girder modelling and stress evaluation utilizing eccentric beams. NUTECH.

Chuan Peng, L., \& Loong Peng, T. (2009). Pipe stress engineering. Houston, Texas: ASME PRESS.

- Garrett, G. (1948).Design of long-span self supporting steel pipe. American Water Works Association Journal, 40(11), 11971206.

Hydraulic Gate and Penstock Association. (1981). Technical standards for gates and penstocks. Japón.

Moss, D., \& Basic, M. (2013). Pressure vessel design manual (4ta ed. inglés). Inglaterra: Elsevier.

Troitsky, M. (1990). Tubular steel structures (2da ed. en inglés). The James Lincoln Arc Foundation.

United States Bureau of Reclamation (USBR). (1986). Engineering monograph No. 3: Welded steel penstocks. Dénver: USBR. 